\title{
La formation des enseignants intervenant en milieu carcéral en France
}

\section{Hugo Barini, Université d'Angers}

\section{Glossaire des abréviations et acronymes}

2CA-SH Certificat complémentaire pour les enseignements adaptés et la scolarisation des élèves en situation de handicap

AP Administration pénitentiaire

CAPA-SH Certificat d'aptitude professionnelle pour les aides spécialisées, les enseignements adaptés, et la scolarisation des élèves en situation de handicap

CECRL Cadre européen commun de référence pour les langues

CEF Centre éducatif fermé

DDL Didactique des langues

DELF Diplôme d'études en langue française

DILF Diplôme initial en langue française

EN Education nationale

EPM Etablissement pour mineurs

FLE Français langue étrangère

FLS Français langue seconde

GÉNÉPI Groupement étudiant national d'enseignement aux personnes incarcérées

MA Maison d'arrêt

RLE Responsable local d'enseignement

SEGPA Sections d'enseignement général et professionnel adapté

\section{Introduction}

L'enseignement en milieu carcéral est un droit. Un droit encadré par des lois européennes et nationales. Un droit auquel tous les détenus, de tous âges et de toutes nationalités ont accès. Un droit universel qui n'est hélas pas reconnu dans tous les établissements pénitentiaires en France. Un droit qui permet aux détenus de recevoir une formation dite « de base », une formation professionnelle, diplômante ou bien une formation langagière pour les détenus allophones. Michel Foucault a écrit sur la prison et a dépeint un espace d'incarcération dans lequel des châtiments corporels étaient exercés pour punir. Alors, on s'est interrogé sur cette question de punition. La prison doit-elle surveiller et punir ou bien peut-elle former, éduquer et instruire ? 
La réinsertion dans la société passe, selon l'Administration pénitentiaire française, par l'enseignement. Le Code pénal dit que «les détenus doivent acquérir ou développer les connaissances qui leur seront nécessaires après leur libération en vue d'une meilleure adaptation sociale » (Section III, article D450). Le domaine de recherche étant principalement l'enseignement du français langue seconde, j'ai choisi de me concentrer sur l'enseignement de cette discipline aux détenus allophones. Si la réinsertion dans la société est synonyme d'acquisition de connaissances pour un détenu francophone, l'enjeu est doublement plus important pour un détenu allophone qui devra apprendre la langue de l'Etat et les codes sociaux qui lui sont propres.

Alors, quels sont les moyens mis en œuvre par l'Etat pour la formation en FLE/FLS (Français langue étrangère/seconde) aux détenus allophones? Une grande partie des établissements carcéraux français proposent aux détenus non-francophones une formation en FLE, dispensés par des enseignants de l'Education nationale (EN) et des bénévoles d'associations. Qui sont donc ces enseignants qui interviennent dans une formation en FLE bien qu'aucun diplôme de l'EN ne concerne l'enseignement de cette discipline - et quelle(s) formation(s) reçoivent-ils ?

Ce présent article a pour objectif de présenter les enseignants intervenant dans le cadre d'une formation en FLE/FLS en milieu carcéral. Il s'agira également d'analyser les différentes formations que ces derniers peuvent recevoir pour répondre au mieux aux besoins du public-détenu non-francophone. Pour terminer ce travail de recherche, j'ai souhaité prendre en compte des professionnels qui ne peuvent pas être acteurs dans la scène carcérale, malgré des compétences en matière de pédagogie non-réfutables : les enseignants titulaires d'un Master DDL - FLE (Didactique des langues - Français langue étrangère).

\section{Recueil des données}

Effectuer une recherche sur cet espace d'enseignement aurait pu se révéler d'une réelle difficulté (demande d'accès à l'établissement, censure de l'administration ou autocensure des détenus (Salane). Néanmoins, ma recherche portait sur la formation des intervenants en milieu carcéral, il n'a donc pas été nécessaire d'observer une classe ou de réaliser des entretiens auprès d'apprenants-détenus. Mes principales sources d'informations étant l'enseignant et les textes scientifiques, j'ai choisi comme méthodes de recueil de données l'entretien individuel et l'analyse de contenus.

Les entretiens individuels ont été menés auprès de différents types d'intervenants en Maisons d'arrêt (MA). Nous avons tout d'abord rencontré deux anciennes bénévoles de l'association GÉNÉPI (Groupement étudiant national d'enseignement aux personnes 
incarcérées). Ces deux étudiantes étaient intervenues dans les ateliers de FLE. Nous sommes ensuite entré en contact avec une enseignante titulaire de l'EN qui, elle aussi, intervenait en MA dans le cadre d'un cours de FLE. Cet entretien, qui s'est tenu en avril 2013, nous a apporté un très grand nombre d'éléments pour la réalisation de notre travail. En effet, le profil de cette enseignante nous a paru intéressant et très représentatif. Enseignante de mathématiques en lycée professionnel, elle a un jour souhaité découvrir un nouveau public et enrichir ses expériences. Ce profil est intéressant dans la mesure où l'enseignante n'a reçu aucune formation en didactique des langues et se retrouve confrontée à un public adulte dans un espace d'enseignement totalement différent du lycée pour y enseigner une discipline qui ne fait pas partie de son champ de compétences. Le fait d'être locuteur francophone suffit-il pour l'enseignement du français à un public non-francophone?

Les entretiens individuels m'ont permis de comprendre et d'analyser les formations reçues par ces intervenants. J'ai également pu prendre conscience des difficultés rencontrées par ces acteurs dans l'exercice de leurs fonctions. Toutefois, cette recherche ne pouvait pas reposer uniquement sur les discours de ces informateurs, car il faut prendre en compte le haut degré de subjectivité de ces témoignages.

\section{L'apprenant-détenu non-francophone : des besoins et objectifs spécifiques}

\subsection{Besoins}

Pour répondre au mieux aux besoins de chaque détenu, il faut d'abord les comprendre. C'est toujours par le biais du test de connaissances et grâce à l'entretien avec le Responsable local d'enseignement (RLE) que l'on va pouvoir établir les besoins de chaque apprenant. On peut toutefois se poser la question du type de formation que l'on va proposer en FLE. Dans ce travail de recherche, nous avons choisi de nous intéresser principalement à la formation en langue française dans le contexte carcéral. Une formation qui est malheureusement parfois confondue avec la lutte contre l'illettrisme, puisque certains détenus francophones ne savent ni lire ni écrire dans leur langue première.

Dans le contexte d'une formation en FLE, quels sont les objectifs à atteindre ? Ce contexte exige de la part de l'enseignant de déterminer rapidement les besoins des apprenants, puisque le problème de la durée de l'incarcération est posé. Il faut prendre en compte que l'on ne connaît pas toujours réellement la durée de la peine et qu'il faut donc essayer de répondre rapidement et efficacement aux objectifs.

Dans une formation en langue française, l'enseignant doit-il favoriser un apprentissage fonctionnel, un apprentissage exclusivement d'ordre linguistique ou une formation davantage 
culturelle en vue d'une réinsertion dans la société ? Michel Febrer explique alors les besoins des apprenants-détenus.

\subsubsection{Besoin fonctionnel}

Dans un établissement pénitentiaire, il est important pour le détenu de pouvoir se tenir au courant de la vie quotidienne. Quelles sont les activités proposées en prison ? Quel est le règlement de l'établissement ? Comment fonctionnent les «bons de cantine » ? Toutes les informations nécessaires pour comprendre la vie quotidienne de l'établissement carcéral passent par l'écrit sur des panneaux d'affichage ou des documents à disposition des détenus. Un détenu allophone doit apprendre ces codes rapidement pour pouvoir vivre son incarcération au mieux et apprendre à devenir indépendant. Il est donc de la responsabilité de l'enseignant d'enseigner le plus rapidement possible aux détenus la lecture et la compréhension des documents par le biais de documents authentiques relatifs à la vie quotidienne des détenus.

\subsubsection{Besoin linguistique}

Le besoin fonctionnel des apprenants-détenus passe obligatoirement par une formation linguistique. Comment lire un règlement intérieur si l'on n'apprend pas à lire et comprendre un document écrit? La compréhension écrite et la production orale sont les principales compétences à développer dans ce contexte de vie. Toutefois, bon nombre de détenus inscrits dans une formation en FLE passent le DILF (Diplôme Initial de Langue Française) ou le DELF (Diplôme d'Études en Langue Française) dans l'objectif de faire valider leurs acquis. Ces certifications valident les quatre compétences linguistiques définies par le Cadre européen commun de référence pour les langues (production orale, compréhension orale, production écrite et compréhension écrite). Pour obtenir cette certification, l'enseignant de langue doit donc faire travailler sa classe sur ces quatre compétences linguistiques.

\subsubsection{Formation culturelle}

Enfin, l'objectif de l'éducation en prison, selon la loi, est avant tout une démarche de réinsertion dans la société française. Trouver un emploi, trouver un logement, vivre une vie ordinaire, toutes ces étapes se font par la langue française. Au-delà donc de la validation de ses compétences, l'apprenant doit aussi anticiper sa libération et sa (ré)insertion. Le cours de FLE permet également aux détenus d'apprendre la culture française. Quels sont les codes à adopter dans la société française ? Certains détenus sont de nouveaux arrivants et ont très peu vécu dans leur pays d'accueil. Il est donc nécessaire de leur faire comprendre les codes de la société, toujours dans une optique de réinsertion. 
De plus, des cours de culture permettent aux apprenants d'échanger sur leur propre culture. Des échanges d'ordre interculturel permettent aux apprenants-détenus de partager leurs expériences, parler de leurs coutumes, de leurs codes. Cela favorise la prise de parole et leur permet de « s'échapper » quelques instants.

\subsection{Objectifs}

Le code de procédure pénale et la loi pénitentiaire de 2009 obligent chaque détenu à exercer une activité, professionnelle ou éducative, au sein de la prison. A son arrivée en centre carcéral, la personne détenue devra passer un test de connaissances générales et de compréhension pour que le RLE puisse évaluer son niveau scolaire et lui proposer une formation adaptée à ses besoins. C'est dans une démarche de repérage de l'illettrisme que les établissements ont mis en place ce dispositif : «Lorsque la personne condamnée ne maîtrise pas les enseignements fondamentaux, l'activité consiste par priorité en l'apprentissage de la lecture, de l'écriture et du calcul. Lorsqu'elle ne maîtrise pas la langue française, l'activité consiste par priorité en son apprentissage » (Loi pénitentiaire du 29 novembre 2009 - Section 2).

Il est important de souligner qu'une grande majorité des détenus n'a passé aucun diplôme, c'est pourquoi il est nécessaire de proposer aux détenus un test de repérage de l'illettrisme. Le RLE doit par conséquent rencontrer chaque détenu pour lui proposer une formation adaptée à ses besoins, et mettre en place celle-ci. Les objectifs pour chaque détenu sont donc personnalisés. Il est presque impossible, dans un tel contexte et dans une telle hétérogénéité de niveaux scolaires, de proposer une seule et même formation aux détenus. L'enseignant doit tenir compte de leurs besoins pour dresser des objectifs spécifiques. Dans certaines formations, il pourra également proposer aux détenus des certifications ou des validations d'acquis. C'est d'ailleurs ces examens qui sont le principal objectif des apprenants, selon l'enseignante de l'EN que nous avons rencontrée. Elle souligne que beaucoup n'ont jamais passé d'examen de leur vie et qu'obtenir une certification est pour eux une fierté personnelle, plus qu'un objectif pédagogique.

\subsection{FLE = lutte contre l'illettrisme ?}

Une des formations mises en place par l'Administration pénitentiaire (AP) et l'EN est la formation langagière en FLE, connexe à la lutte contre l'illettrisme. Dans cette démarche, les deux administrations proposent à chaque détenu un entretien pendant lequel le RLE pourra constater le niveau de connaissances langagières de la personne. Si ses connaissances sont jugées trop faibles, le détenu, francophone ou allophone, pourra suivre une formation appropriée. 
Christiane Cavet définit le terme illettrisme de la façon suivante :

La France, en adoptant le mot « illettrisme », a choisi de distinguer deux situations : l'illettrisme pour les personnes francophones scolarisées en langue française, qui n'ont pas acquis une maîtrise suffisante de la communication écrite; et l'analphabétisme pour les personnes étrangères, ou d'origine étrangère, n'ayant jamais appris un code écrit. Si dans les deux cas, il y a bien une absence ou une insuffisance de la capacité à lire et à écrire en langue française, les causes du problème, le vécu des personnes, et les modalités d'accompagnement diffèrent. Ainsi est née la lutte contre l'illettrisme qui s'est développée à côté de la politique de formation linguistique des migrants. (13)

C'est en 2010 que le dispositif de lutte contre l'illettrisme en milieu carcéral a vu le jour. En 2011, 124 MA sur les 133 ont mis ce dispositif en place pour couvrir 98\% de la population carcérale entrante. Les tests de repérage se présentent sous la forme d'un entretien oral, puis d'un «bilan lecture ». En décembre 2011, on estimait le pourcentage de détenus ayant des difficultés en français à $14 \%$ de la population carcérale. On peut alors dissocier deux catégories : les apprenants n'ayant pas assez de connaissances en langue orale pour passer le test de lecture (souvent les personnes non-francophones) et les détenus ayant une connaissance « rudimentaire » de la langue orale, mais peu sur la langue écrite.

Dans tous les cas et dès lors que ces personnes détenues ne réussissent pas le test de lecture, le RLE leur propose une formation de base, ou de FLE (français langue étrangère) pour les individus non-francophones. L'AP qualifie une personne non-francophone comme étant une personne dont le niveau de langue française ne répond pas aux compétences exigées par le DILF.

Notons cependant que les apprenants-détenus ne suivent parfois pas les conseils donnés par le RLE concernant la formation qui leur serait appropriée. En effet, dans le rapport de l'AP, nous remarquons que $1,4 \%$ de la population carcérale en situation d'illettrisme choisit de suivre une formation professionnelle et non pas une formation de base ou de remise à niveau. Dans les recommandations faites dans le rapport de l'AP, une d'entre elles concerne la difficulté de « distinguer strictement un public non francophone qui relèverait des actions de FLE d'un public illettré qui relèverait des actions d'alpha-illettrisme ». Le terme FLE dans ce contexte est flou, car il ne désigne qu'une population carcérale non-francophone dont les connaissances en français sont très faibles, voire nulles. Qu'en est-il alors des apprenants détenus non francophones, dont le niveau en langue française est intermédiaire ? Ce type de formation relève-t-il alors du champ du FLE ou juste d'une remise à niveau ? 


\section{Les profils d'enseignants de FLE intervenant en milieu carcéral}

\subsection{Enseignant titulaire de l'Éducation nationale}

C'est dans la convention et la circulaire du 29 mars 2002, signées par l'Administration pénitentiaire et le Ministère de l'éducation, que le dispositif d'enseignement en milieu carcéral est défini. Cette convention établit les bases de l'enseignement dans ce contexte bien que l'établissement carcéral soit le seul décisionnaire des enseignements proposés dans ses locaux. Le Ministère de l'éducation est en charge d'affecter des enseignants dans ces établissements. Ce sont les enseignants titulaires d'un diplôme de l'EN qui interviennent auprès des prévenus des MA, des détenus des centres de détention ou des jeunes délinquants des Centres éducatifs fermés (CEF) et des Établissements pénitentiaires pour mineurs (EPM). Selon le rapport de l'AP, pour l'année 2011-2012, on comptait 466,5 postes enseignants à temps plein intervenant dans le contexte carcéral (la plupart d'entre eux sont des enseignants du premier degré). Il faut toutefois ajouter à ce chiffre les enseignants intervenant dans le cadre de vacations, soit l'équivalent de 233,5 postes à temps plein.

Dans son témoignage, Patrick Leterrier nous explique que c'est par intérêt pour le public adulte qu'il choisira d'intervenir en MA (5). Il explique également son parcours et regrette que peu d'enseignants de l'EN choisissent cette voie par manque d'information sur ce public ou à cause des préjugés sur cet environnement de travail particulier. Lui n'est pas un enseignant spécialisé, mais c'est grâce à ses expériences antérieures auprès de publics adultes qu'il obtiendra le poste d'enseignant à la MA de Cherbourg.

\subsection{Bénévole d'associations}

Malgré l'effort de l'EN pour permettre aux détenus un accès à l'éducation, il existe toujours un réel manque d'intervenants pour l'enseignement en milieu carcéral. La présence d'associations pour pallier ce manque est donc indispensable. La principale est Auxilia, qui offre aux détenus des formations à distance et concerne environ $50 \%$ de la population carcérale scolarisée. L'offre de formation est très diversifiée et quasi gratuite. Les enseignants bénévoles sont pour la plupart des enseignants de l'EN à la retraite.

Le GÉNÉPI est une autre association majeure pour la réinsertion et l'éducation des personnes détenues. Il offre aux détenus des activités variées et des enseignements en présentiel. Les étudiants qui interviennent en milieu carcéral, sont issus de formations supérieures et offrent aux détenus allophones des ateliers de FLE. L'association permet 
également de sensibiliser la population aux conditions de vie dans les prisons en animant des évènements dans les universités ou dans des centres culturels.

Ces associations sont toujours sous la tutelle du Ministère de l'éducation et les enseignements se font en collaboration avec les RLE des établissements carcéraux.

\section{Les formations reçues par les enseignants intervenant en milieu carcéral}

\subsection{Formation de terrain}

Tout comme le préconise la Convention signée entre l'EN (Education nationale) et l'AP, des formations doivent être proposées aux enseignants intervenant dans un établissement carcéral pour familiariser ces derniers au contexte d'enseignement en prison. Je me suis donc intéressé à la formation dispensée par l'AP et à la formation reçue par les bénévoles de l'association GÉNÉPI. Ces étudiants bénévoles ne sont pas tous issus d'une formation en didactique et n'ont, pour la plupart, jamais enseigné. Le contenu de la formation délivrée par l'association est-elle alors en lien avec l'enseignement ou le contexte ? J'ai choisi d'appeler ces deux types de formation les formations « de terrain ».

\subsubsection{Formation GÉNÉPI}

Les bénévoles de l'association GÉNÉPI peuvent assister à plusieurs sessions de formation délivrées par des formateurs de l'association. Ces formations ont lieu plusieurs week-ends dans l'année et permettent ainsi d'informer et d'échanger sur les pratiques dans le contexte carcéral. L'association GÉNÉPI étant gérée localement par des étudiants bénévoles, il m'a été impossible d'entrer en contact avec des responsables de formation. Je me suis donc appuyé sur les informations recueillies par d'anciennes bénévoles de l'association pour relever les différentes composantes de la formation GÉNÉPI.

\section{a) Informer}

Un premier week-end de formation est proposé à tous les intervenants bénévoles souhaitant animer des activités en milieu carcéral. Les activités offertes par l'association étant très variées (atelier de code de la route, jeux de société, théâtre, FLE, etc.), le profil des bénévoles est lui aussi diversifié. Une formation de base leur apporte plusieurs connaissances sur divers axes :

- les missions et actions de l'association,

- la présentation générale du milieu carcéral,

- le profil du public. 
C'est sous la forme de plusieurs ateliers et de tables rondes que cette formation se déroule. Les nouveaux «génépistes» (membres de l'association GÉNÉPI) peuvent alors poser des questions à des formateurs ou aux bénévoles plus anciens dans l'association pour s'informer davantage sur le contexte et l'association.

\section{b) Rassurer}

La présence de formateurs et d'anciens bénévoles est nécessaire pour rassurer les nouveaux intervenants. Dans un contexte aussi singulier, intervenir en tant que bénévole peut être parfois effrayant. Les ateliers permettent alors des échanges avec les pratiques des anciens, ce qui permet de conseiller et de rassurer.

Toutefois, comme l'a fait remarquer une informatrice bénévole de l'association lors de notre entretien, bien que la formation permette un échange avec d'autres bénévoles, chaque établissement pénitentiaire est différent : les relations avec le personnel peuvent varier d'un établissement à l'autre et il en est de même avec les détenus. Certains établissements feront intervenir des génépistes pour assurer des ateliers de FLE à un public d'hommes alors que d'autres proposeront des ateliers à des femmes. Tous ces facteurs rendent les interventions très différentes les unes des autres. Il est parfois possible que plusieurs bénévoles de l'association soient affectés dans le même établissement alors que les MA les moins importantes n'auront besoin que d'un génépiste. Notre informatrice insiste alors sur le fait que la formation délivrée par le GÉNÉPI est intéressante et nécessaire pour connaître le contexte d'intervention, mais qu'elle ne répond pas vraiment aux besoins réels puisqu'il « fallait vraiment se lancer pour voir comment ça allait se passer ».

\section{c) Prévenir}

Lors de ces week-ends de formation, les échanges sont favorisés entre les intervenants pour rassurer les novices mais aussi dans un but préventif. Dans un contexte qui peut parfois faire peur, il est nécessaire d'avertir les nouveaux bénévoles de situations qui peuvent créer certaines difficultés. Les relations interprofessionnelles peuvent parfois engendrer des difficultés. Il est alors nécessaire de conseiller les nouveaux génépistes sur la façon d'agir en contact des membres du personnel pénitentiaire. De plus, c'est en s'appuyant sur les situations vécues que les formateurs du GÉNÉPI peuvent illustrer des situations difficiles. Mon informatrice donne notamment l'exemple d'un cas dans lequel un étudiant-détenu demande à l'intervenant de transmettre un mot à un membre de sa famille. De telles situations peuvent dérouter l'intervenant, qui ne doit pas, rappelons-le, être un relais entre le détenu et des personnes extérieures. 


\subsubsection{Formation de l'AP}

La formation délivrée par l'AP est appelée «formation d'adaptation à l'emploi ». Cette formation est obligatoire. Quel en est le contenu ? Je me suis appuyé sur la Convention signée entre l'EN et l'AP pour y répondre. L'entretien individuel que j'ai mené avec une enseignante titulaire de l'EN, me permet également d'y ajouter quelques précisions.

\section{a) Contexte carcéral général}

Avant d'intervenir en contexte carcéral, mon informatrice n'avait reçu aucune formation. Une visite détaillée lui avait été proposée par le RLE qui en avait profité pour lui expliquer le fonctionnement de l'établissement. Cette visite a permis à la nouvelle intervenante de se familiariser avec le contexte fermé, un milieu qui avait toujours suscité une certaine curiosité : «J'habite à côté de la maison d'arrêt, je passe devant quotidiennement et j'ai toujours été un petit peu intriguée par ce lieu là... ce lieu fermé » (Propos recueillis lors d'un entretien avec une enseignante titulaire de 1'EN, avril 2012). Un tour des locaux dans leur intégralité permet aussi aux intervenants de se rendre compte et de comprendre le quotidien des détenus. Une présentation avec les membres du personnel pénitentiaire a également été nécessaire.

C'est après un mois d'intervention qu'une réunion d'une matinée a été proposée par un membre de l'AP. Cette réunion avait pour objet d'expliquer davantage aux intervenants extérieurs le monde carcéral en leur expliquant le fonctionnement de la MA, mais aussi d'autres types d'établissements pénitentiaires, comme les EPM et les Centres de détention. Les missions de l'AP ont aussi été décrites et certains termes juridiques tels que « remise de peine » ont été explicités. Le fonctionnement de l'établissement pénitentiaire est un élément primordial qui peut énormément influer dans l'espace-classe.

\section{b) Formation à partir de l'expérience de l'enseignant}

Cette formation délivrée par l'AP a aussi permis aux intervenants de poser des questions sur des situations particulières. Il est en effet intéressant et très pertinent de proposer une telle formation après un mois d'interventions dans l'établissement. Les enseignants ont vécu des situations qui peuvent être difficiles ou qu'ils ne comprennent pas. Cette formation permet à l'enseignant de mieux comprendre ces situations particulières. C'est alors un échange entre les enseignants et l'AP sur des cas concrets. Les intervenants peuvent expliquer des situations précises auxquelles ils ont dû faire face. Les apprenants-détenus voient aussi l'enseignant comme un relais puisqu'ils sont en contact direct avec eux et car la parole est parfois plus libérée qu'avec les membres du personnel pénitentiaire. Il est courant que les détenus s'adressent aux enseignants pour demander des conseils sur des situations 
administratives. La formation a donc pour but de renseigner au mieux les intervenants pour que ces derniers soient dans la capacité de répondre aux interrogations des détenus.

\section{c) Suivi professionnel}

Comme pour les enseignants spécialisés, les enseignants intervenant en milieu carcéral peuvent être inspectés par l'inspection académique. Une observation de classe est suivie par un entretien dans lequel l'enseignant peut parler de ses difficultés et de son projet professionnel. L'Inspecteur de l'EN se doit alors de proposer à l'enseignant, en fonction de son profil et de ses objectifs, des formations pour favoriser une dynamique de carrière. La Convention dit que « en raison des spécificités de l'enseignement en milieu pénitentiaire, la nécessité de participer à des actions de formation continue doit être rappelée à tous les enseignants dans le cadre de cet entretien » (décembre 2011, 10).

Dans le cadre de l'entretien mené avec l'enseignante intervenant en MA, j'ai insisté sur la formation reçue. Il est apparu que la formation reçue sur l'environnement de travail correspondait assez bien aux attentes de l'enseignante. Toutefois, l'enseignante est titulaire d'un diplôme lui permettant d'enseigner les mathématiques en lycée professionnel. En MA, elle donne des cours de FLE. Elle insiste alors, dans l'entretien, sur le manque de formation sur l'objet d'apprentissage, dans ce cas, le français langue étrangère. Aucun document officiel ne mentionne la formation des enseignants à la discipline enseignée. Toutes les formations proposées portent sur l'environnement de travail particulier ou sur le public spécifique. Les difficultés des enseignants en contexte carcéral concernent effectivement le public et le contexte, mais également la matière qu'ils y enseignent sans avoir jamais reçu de formation appropriée. Encore une fois, nous pouvons mentionner le «bricolage pédagogique » auquel les enseignants doivent avoir recours dans le but de pallier une certaine «insécurité pédagogique $»$.

Les formations reçues par les bénévoles et enseignants intervenant en contexte carcéral concernent principalement l'environnement et le public. Néanmoins, dans les deux cas, aucune formation sur la pédagogie ou la didactique d'une langue étrangère n'est proposée. Il est assez courant que le GÉNÉPI confie des ateliers de langue française pour apprenants non-francophones ou en situation d'illettrisme à des étudiants qui n'ont jamais reçu de formation pour l'enseignement. Les formations d'un week-end n'apportent aucun outil pédagogique. Il est alors de la responsabilité du RLE de l'établissement de former rapidement le génépiste à des situations d'enseignement.

L'enseignant intervenant en milieu carcéral a, quant à lui, l'expérience nécessaire dans l'enseignement pour faire face à des situations de difficultés d'apprentissage. Toutefois, ces 
postes sont pourvus par des enseignants du premier ou second degré ${ }^{1}$. Le public d'un établissement pénitentiaire étant majoritairement un public adulte, les besoins sont différents. De plus, certains enseignants se voient confier l'enseignement d'une discipline qui n'est pas dans leur champ de compétences. L'exemple de l'enseignante de mathématiques en lycée professionnel est représentatif. Une formation pour l'enseignement d'une discipline serait alors appréciée puisque ce genre de situations peut créer un sentiment d'insécurité pour l'enseignant, un sentiment d'autant plus important que l'environnement et le public sont encore inconnus de l'enseignant.

Alors, quelle formation répond le mieux aux besoins d'un enseignant de FLE en milieu carcéral ? La formation en didactique des langues proposée par les départements de FLE des universités françaises permet d'acquérir des connaissances sur les méthodes pédagogiques adaptées à l'enseignement de langues étrangères et apportent également des connaissances sur les besoins et objectifs de publics variés (étudiants en mobilité, migrants, enfants, adolescents, etc.). Il nous paraît intéressant, pour terminer, de décrire les compétences des enseignants de FLE et enfin d'analyser les représentations de ces derniers concernant l'enseignement du FLE en milieu carcéral.

\subsection{Formation spécialisée}

Selon la Convention entre l'EN et le Ministère de la justice et des libertés (décembre 2011, 10), «le critère de recrutement [d'un enseignant en établissement pénitentiaire] est l'expérience pédagogique acquise antérieurement à des postes d'enseignement spécialisé, auprès de mineurs en difficulté, dans la formation pour adultes ou dans des établissements difficiles ». Cette clause de la convention définit donc l'expérience d'un enseignant selon deux critères d'expériences professionnelles : le type d'établissement et le type de public. Il faut noter qu'une majorité des enseignants intervenant en milieu carcéral sont des enseignants spécialisés, titulaires d'une certification de l'éducation nationale option F. Il s'agit à présent d'expliciter les objectifs de cette formation initiale et d'en dégager les compétences acquises par l'enseignant. Le rôle et la formation du RLE sont également définis dans la Convention.

\subsubsection{Présentation et objectifs des formations spécialisées}

La formation délivrée par le Ministère de l'éducation nationale est une formation de spécialisation pour les enseignants titulaires d'un diplôme d'enseignement. La formation intitulée « Certificat d'aptitude professionnelle pour les aides spécialisées, les enseignements adaptés, et la scolarisation des élèves en situation de handicap » (CAPA-SH) est destinée aux enseignants du premier degré et a pour but de les former à enseigner à des publics en difficulté. La formation pour les enseignants du second degré est appelée 2CA-SH « Certificat 
complémentaire pour les enseignements adaptés et la scolarisation des élèves en situation de handicap ». Cette dernière ne permet pas la titularisation des enseignants sur des postes d'enseignants spécialisés. Ces postes sont pourvus par des enseignants du premier degré qui sont souvent affectés dans des établissements scolaires dits «difficiles » ou dans des classes spécialisées (les Sections d'Enseignement Général et Professionnel Adapté [SEGPA] par exemple). Les postes dans ce type d'établissements doivent donc être pourvus par des enseignants titulaires du CAPA-SH. Les enseignants du second degré doivent dans tous les cas être en mesure d'accueillir dans leur classe des élèves en difficulté ou en situation de handicap, bien que leur statut institutionnel ne leur permette pas d'être enseignants spécialisés.

Ces deux formations sont complétées par des options de spécialisation qui permettent l'approche de différents publics ou différentes structures. Pour répondre au mieux aux besoins des apprenants-détenus, les enseignants spécialisés intervenant en milieu carcéral ont suivi une formation CAPA-SH ou 2CA-SH option $\mathrm{F}$ «Enseignants spécialisés chargés de l'enseignement et de l'aide pédagogique auprès des élèves des établissements d'enseignement général et professionnel adapté ». A défaut d'une formation pour l'enseignement auprès de publics adultes, cette option dans la formation spécialisée est celle qui répond au mieux aux besoins des détenus et à leurs objectifs de réinsertion.

Pour mieux comprendre les objectifs de cette option, je me suis appuyé sur le Bulletin Officiel $^{2}$ (BO) du 30 avril 2009, bien que cette publication ne mentionne que l'enseignement en SEGPA. Il est donc nécessaire de faire un parallèle entre les deux types de structures : la SEGPA et la prison. Le BO classe les objectifs de la formation des enseignants spécialisés option F selon trois axes :

\section{a) Principes d'organisation pédagogique :}

Ce premier axe traite principalement des démarches pédagogiques qui prennent en compte les difficultés des apprenants. Il préconise une individualisation des élèves et un accompagnement personnalisé pour recréer un climat de confiance entre l'élève et l'École. Le parcours scolaire des élèves en SEGPA (et des apprenants-détenus) a souvent été difficile et il est indispensable que l'enseignant puisse leur redonner le goût de l'apprentissage en utilisant, par exemple, la pédagogie de projet comme méthode. Cette méthode, qui implique davantage l'apprenant, est adaptée à un public difficile, puisqu'elle responsabilise les étudiants et les rend acteurs de leur apprentissage. Toutefois, l'enseignant spécialisé doit aussi être capable de proposer à chaque élève un projet individuel de formation pour lui permettre de répondre à ses besoins et attentes. Un suivi pédagogique et une concertation de l'équipe enseignante sont 
indispensables pour ajuster ce projet. La formation des élèves en SEGPA a également pour but l'obtention du Certificat de formation générale (CFG). Tout comme en milieu carcéral, la réussite à cet examen est un réel facteur de motivation chez les apprenants. L'enseignant spécialisé doit alors proposer des exercices de préparation à cet examen. En ce qui concerne l'enseignement en SEGPA, le BO insiste enfin sur l'accès à la formation professionnelle en proposant aux élèves des stages d'observation en milieu professionnel et la mise en place d'un «plateau technique», espace d'information au sein de l'établissement permettant la découverte des différents champs professionnels.

\section{b) Préparation à l'accès à une formation professionnelle}

La pratique en milieu professionnel fait l'objet d'un des trois objectifs de la formation spécialisée option F. En milieu carcéral, la formation professionnelle peut être mise en place par des organismes divers. L'enseignant spécialisé n'aura pas à introduire l'accès à la formation professionnelle dans son programme, contrairement aux enseignants spécialisés intervenant en SEGPA.

\section{c) Acquisition du socle commun}

Comme tout établissement du Second degré, les SEGPA se doivent d'enseigner des compétences nécessaires à l'acquisition du «socle commun» de compétences. Ce terme désigne un niveau de connaissances et un ensemble de compétences qu'un élève doit avoir acquis au terme de sa scolarité obligatoire. Un programme doit être défini par l'équipe pédagogique pour répondre aux objectifs du socle. Ces objectifs sont similaires aux objectifs d'une formation en milieu carcéral, selon les niveaux d'apprentissage. Ils comprennent une connaissance de la langue française (lue, écrite et parlée), la maîtrise du niveau A2 d'une langue étrangère vivante, des éléments de mathématiques et de culture scientifique et enfin l'acquisition de compétences civiques, humanistes et sociales. Ces compétences transversales peuvent être considérées comme des compétences visant la réinsertion sociale et professionnelle des jeunes en difficulté dans le cas d'une SEGPA, et des détenus dans le contexte carcéral. Elles ont comme objectifs d'apporter un regard critique aux apprenants, une curiosité, de les sensibiliser aux règles de sécurité et aux règles de fonctionnement d'un établissement et d'un état. En milieu carcéral, cela est mis en place par des cours de culture visant à présenter les codes et coutumes de la France aux détenus étrangers par exemple, ou par le biais d'ateliers visant la réinsertion, tels que l'apprentissage du code de la route.

Ces objectifs, bien que définis dans le cadre d'un enseignement en SEGPA, répondent presque complètement aux objectifs pédagogiques d'un enseignement en milieu carcéral. Les autres formations spécialisées mises en place par l'EN répondent à des besoins de formation 
pour l'enseignement de publics en situation de handicap. L'option F des formations CAPASH et 2CA-SH est alors la plus appropriée dans un contexte d'enseignement en milieu carcéral, bien qu'elle soit mise en place pour des jeunes en difficulté et non pas pour un public adulte. Contrairement aux autres options qui sont définies par un type de public (élèves sourds ou malentendants, aveugles ou malvoyants, etc.), l'intitulé de l'option F passe par une définition de la structure, alors que cette formation ne concerne pas uniquement les enseignants spécialisés de SEGPA. Un intitulé qui engloberait les deux types de publics (jeunes et adultes) et les différents types de structures (SEGPA, établissement pénitentiaire) serait alors plus pertinent.

\subsubsection{Conditions de recrutement d'un enseignant}

La convention signée entre le Ministère de l'éducation nationale et l'AP définit dans son annexe 2 les conditions de nomination des enseignants souhaitant intervenir en milieu carcéral. Comme mentionné au début de cet article, le recrutement se fait selon les expériences pédagogiques de l'enseignant dans des établissements dits « difficiles » ou auprès de jeunes en difficulté ou d'adultes. Il est important de relever que les enseignants qui choisissent d'enseigner dans un établissement carcéral restent titulaires de leur précédent poste pendant un an, dans le but de leur permettre une adaptation à ce contexte d'enseignement particulier. En effet, outre la motivation témoignée par les enseignants, une période d'adaptation dans ce contexte est nécessaire car il arrive que l'environnement et le public ne correspondent pas aux attentes ou aux compétences de l'enseignant. L'enseignant intervenant en milieu carcéral recevra une rémunération supplémentaire qui a pour but « de compenser les sujétions particulières liées aux conditions d'exercice en milieu pénitentiaire » (décembre 2011, 10).

Un suivi professionnel de l'enseignant est également mis en place lors des inspections pédagogiques. Elles ont pour but de faire le point sur sa démarche pédagogique et de discuter des difficultés auxquelles il peut être confronté. Des formations mises en place par 1'EN peuvent être proposées, dont la formation pour devenir enseignant spécialisé (CAPA-SH, 2CA-SH). Ces formations continues ont aussi pour objet de répondre aux attentes de l'enseignant et permettre une évolution de carrière.

\subsubsection{Contenu des formations spécialisées et compétences de l'enseignant}

L'arrêté du 5 Janvier 2004 et le Bulletin Officiel 4 du 26 février 2004 définissent de façon précise le contenu de la formation CAPA-SH pour l'option F. La formation CAPA-SH, destinée aux enseignants du premier degré est une formation en alternance. Une année de formation est donc partagée entre un poste à mi-temps dans un établissement spécialisé et des 
sessions de formations. La première session est une formation de base et a pour but de former les enseignants aux besoins particuliers du public. Elle dure en moyenne trois semaines et se déroule souvent au mois de mai ou juin précédant la prise de poste spécialisé. Durant l'année scolaire, les enseignants en formation auront d'autres sessions de formation d'une durée maximale d'une semaine chacune. Le contenu de ces formations théoriques est réparti selon trois unités :

- Unité de formation 1: «Pratiques pédagogiques différenciées et adaptées aux besoins des élèves ». Cette unité de formation consiste en l'analyse des besoins spécifiques aux élèves des établissements spécialisés. Elle exige une bonne connaissance des besoins de l'enfant et de l'adolescent, de leur développement et apporte des outils pour le repérage des difficultés d'apprentissage. Cette unité permet aussi d'affiner ses connaissances sur la méthodologie de l'observation et de l'évaluation des élèves pour pallier leurs difficultés et ainsi proposer une démarche pédagogique adaptée « dans une perspective de prévention ou de remédiation ».

- Unité de formation 2: «Pratiques professionnelles au sein d'une équipe pluricatégorielle ». Cette unité a pour objectif de familiariser l'enseignant à une collaboration entre les différents corps professionnels présents dans un établissement spécialisé afin de proposer aux élèves des projets éducatifs et professionnels. Elle a aussi pour mission d'apporter les connaissances suffisantes à l'enseignant spécialisé sur son rôle et ses tâches.

- Unité de formation 3 : «Pratiques professionnelles prenant en compte les données de l'environnement familial, scolaire et social ». Cette dernière unité de formation apporte des connaissances sur les fondements de l'enseignement spécialisé dans la législation française, mais aussi dans le contexte européen et mondial. Elle consiste aussi à donner des définitions aux termes de handicap, difficulté scolaire et éducation.

Notons finalement que les contenus des formations CAPA-SH et 2CA-SH sont assez similaires. La formation CAPA-SH développe toutefois davantage l'unité de formation sur la collaboration entre les équipes.

Il est alors nécessaire de s'interroger maintenant sur la pertinence de cette formation pour l'enseignement en milieu carcéral. Ces trois unités de formation répondent-elles vraiment aux besoins d'un enseignant qui souhaite enseigner en prison ? Les principaux axes de la formation reposent encore une fois sur un public jeune. Des connaissances sur le développement de l'enfant sont effectivement nécessaires si l'on souhaite enseigner dans une SEGPA. Toutefois, les besoins d'un adolescent en difficulté restent assez éloignés des besoins d'un détenu. Il s'agit aussi de différencier ces deux contextes d'enseignement par les équipes 
professionnelles qui composent les établissements. Dans un contexte de MA par exemple, le RLE est en contact permanent avec le directeur de l'établissement et avec l'AP. Les enseignants, quant à eux, ne sont qu'en contact indirect avec ces acteurs, leur unique référent étant le RLE. Le projet pédagogique et les démarches sont des choix effectués par le RLE en concertation avec l'Unité Pédagogique Régionale.

Néanmoins, cette formation délivrée par l'EN est celle qui peut correspondre le mieux aux besoins de ces apprenants-détenus puisqu'elle répond à des problématiques d'insertion et de difficultés d'apprentissage même si elle ne s'adresse pas aux mêmes types de publics et que son intitulé reste vague.

\section{L'enseignant titulaire d'un Master FLE : quelles compétences pour quels besoins ?}

Titulaire d'un Master «Didactique des langues, français langue étrangère et technologies éducatives », j'ai choisi de décrire le contenu de cette formation pour mettre en exergue les compétences acquises nous permettant d'enseigner le FLE. Cette formation universitaire est une formation propre à une université française. La liste des compétences travaillées dans ce cursus est une liste non-exhaustive, puisque chaque université française établit un programme différent.

Les compétences à acquérir dans une formation initiale en didactique des langues concernent deux axes principaux : devenir un enseignant et enseigner une langue étrangère. Au cours de sa formation, un étudiant en Didactique des langues (DDL) - FLE devra prendre connaissance des différents courants pédagogiques et devra être capable de concevoir un programme et des activités. De plus, l'étudiant en FLE découvrira dans sa formation différents profils d'apprenants grâce à l'intervention de professionnels ou des stages. Cette diversité dans les publics permet de développer les compétences et d'asseoir les connaissances théoriques dans un contexte pratique.

\subsection{Devenir enseignant}

Les compétences comprennent d'abord l'acquisition d'un cadre théorique concernant les politiques éducatives, les sciences de l'éducation et les sciences du langage. Afin de mettre en pratique ces connaissances, les étudiants seront amenés à effectuer un stage.

\subsubsection{Acquisition d'un cadre théorique}

L'acquisition d'un cadre théorique a pour objet de prendre connaissance des différentes pratiques pédagogiques. Les enseignements théoriques sont davantage enseignés lors de la première année de Master. Cela permet aux étudiants d'apporter un regard critique 
sur les différentes méthodes d'enseignement et les former au métier d'enseignant. Des travaux sur l'acquisition des langues sont alors nécessaires pour réussir à concevoir des activités pédagogiques capables de répondre aux besoins de chaque apprenant.

La seconde année de formation apporte un sens de l'analyse par la réalisation d'un travail de recherche. Des unités d'enseignements telles que «Méthodologie de recherche en éducation » et « Méthodologie du travail de recherche » permettent une analyse plus profonde de situations et de pratiques éducatives.

\subsubsection{Stages pratiques et interventions de professionnels}

Pendant ses deux années de formation dans cette université, l'étudiant en DDL-FLE devra effectuer deux stages (un court et un long) pour lui permettre de s'impliquer professionnellement dans un contexte d'enseignement. Ces expériences sont, pour la plupart des étudiants, une première entrée dans le métier d'enseignant. Chaque stage doit être complété par la rédaction de rapports apportant une réflexion sur la pratique professionnelle. Les stages peuvent être effectués dans différentes structures et les rapports sont soutenus face à un jury composé d'enseignants ou de professionnels dont les compétences sont en adéquation avec le sujet traité.

Enfin, de nombreuses rencontres avec des intervenants extérieurs permettent aux étudiants de prendre conscience des différentes facettes de la profession. En fonction des publics et en fonction des structures, la pratique professionnelle peut varier. Ces témoignages extérieurs permettent alors de connaître des champs professionnels variés, de créer de nouvelles envies ou de nouvelles ambitions. Les intervenants sont des professionnels aux parcours variés et leur témoignage peut apporter de nouvelles perspectives dans la vision des étudiants sur le métier de l'enseignant.

La diversité des profils des professionnels intervenant dans la formation initiale d'un étudiant est une richesse, dans la mesure où elle ouvre le champ des possibilités. En effet, les étudiants découvrent, lors de leur formation, une diversité dans les pratiques professionnelles qui leur permettent alors de définir leurs projets professionnels. Toutefois, devenir enseignant ne se résume pas uniquement au savoir-être enseignant, c'est aussi enseigner une discipline, une langue étrangère.

\subsection{Enseigner une langue}

\subsubsection{Réflexion sur la langue}

Dans la formation universitaire en DDL-FLE que j'ai suivie, une très grande majorité des étudiants ont comme première langue le français. L'une des difficultés ressenties par ces futurs-enseignants est alors de savoir enseigner sa première langue comme une langue 
étrangère ou seconde. Les processus d'acquisition d'une première langue sont différents de l'acquisition d'une langue seconde. La formation initiale reçue par les étudiants du Master permet donc une prise de recul sur la langue française et apporte des outils indispensables à l'enseignement de celle-ci.

\subsubsection{Outils d'enseignements et évaluation}

C'est dès la première année de formation que les enseignements apportant une réflexion sur la langue sont proposés. Des unités d'enseignement (UE) telles que «Grammaire et enseignement des langues» ou « de la description du français à son enseignement» permettent de redécouvrir sa langue première, dans le but de l'enseigner comme une langue étrangère. Ces enseignements apportent également de nouveaux outils dans la conception d'une séquence pédagogique et pour le déroulement d'activités de classe. Des mises en situation sont aussi proposées aux étudiants de la formation, afin de mettre en contexte et en pratique ces nouveaux outils.

Enseigner, c'est aussi savoir évaluer. Afin d'être capable de proposer aux apprenants un programme répondant aux objectifs des apprenants, il est indispensable de pouvoir les cibler. Des enseignements sur la conception d'outils d'évaluation sont alors proposés aux étudiants en FLE. Ces UE apportent aussi des connaissances sur les différents types d'évaluation et les certifications en langue française.

\subsubsection{Découvrir différents publics}

Cette formation en DDL-FLE comprend des unités d'enseignements au choix pour offrir aux étudiants la possibilité de découvrir des publics différents. Selon chaque public, les besoins sont définis, ainsi que les objectifs. Il est alors intéressant de découvrir des outils pédagogiques pour répondre aux objectifs spécifiques de ces publics. Au choix, quatre publics : migrants, professionnels, étudiants en mobilités ou apprenants en situation d'illettrisme. Ces unités d'enseignement sont dispensées par des professionnels ayant exercé dans des structures concernées.

Ce Master DDL-FLE permet ainsi aux futurs étudiants de découvrir des publics qui peuvent leur être inconnus dans le but de concevoir un projet professionnel pour l'avenir, mais aussi de se construire une "mallette pédagogique » pour enseigner la langue française dans différentes structures. Le Master 2 apporte davantage de connaissances sur un public adulte, alors que le Master 1 répond aussi aux besoins de l'enfant et à l'acquisition des langues en général.

Les enseignants intervenant en milieu carcéral reçoivent tous une formation, qu'elle soit d'ordre théorique ou pratique, sur l'enseignement dans ce contexte particulier. Toutefois, 
l'enseignant titulaire d'un Master DDL-FLE ne peut exercer ses fonctions entre les murs d'un établissement pénitentiaire. Bien qu'il ait les connaissances nécessaires relatives à l'enseignement d'une langue seconde à un public adulte, l'enseignant de FLE ne peut travailler en prison, car les accords signés entre l'AP et le Ministère de l'éducation exigent des enseignants soient titulaires d'un diplôme de l'EN.

Ces accords ne prennent aucunement en considération les enseignants titulaires d'un Master DDL-FLE bien que leur niveau d'études et leurs compétences professionnelles soient équivalentes.

Aucune des formations détaillées dans cet article ne correspond réellement et en totalité aux besoins et aux objectifs d'un apprenant-détenu non francophone. Il faut prendre en compte plusieurs éléments, tels que l'enseignement à un public adulte, non-francophone, dans un contexte particulier. Chacune de ses formations doit être améliorée et complétée.

Tableau récapitulatif des formations

\begin{tabular}{|c|c|c|c|c|c|}
\hline & & $\begin{array}{c}\text { Formation } \\
\text { CAPA-SH } \\
\text { Option F }\end{array}$ & $\begin{array}{l}\text { Formation } \\
\text { GÉNÉPI }\end{array}$ & $\begin{array}{c}\text { Formation } \\
\text { Administration } \\
\text { Pénitentiaire }\end{array}$ & $\begin{array}{c}\text { Formation } \\
\text { Didactique des } \\
\text { Langues }\end{array}$ \\
\hline \multirow{3}{*}{ 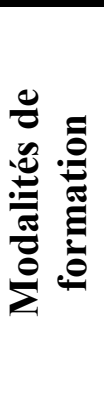 } & $\begin{array}{l}\text { Type de } \\
\text { formation }\end{array}$ & $\begin{array}{l}\text { Formation } \\
\text { continue }\end{array}$ & $\begin{array}{c}\text { Ateliers } \\
\text { d'information }\end{array}$ & $\begin{array}{c}\text { Réunion } \\
\text { d'information }\end{array}$ & Formation initiale \\
\hline & $\begin{array}{l}\text { Durée de la } \\
\text { formation }\end{array}$ & Un an & $\begin{array}{c}\text { Deux week- } \\
\text { ends / an }\end{array}$ & Une matinée & Deux ans (Master) \\
\hline & Public ciblé & $\begin{array}{c}\text { Enseignants } \\
\text { éducation } \\
\text { nationale }\end{array}$ & Etudiants & $\begin{array}{c}\text { Enseignants } \\
\text { Education } \\
\text { Nationale }\end{array}$ & $\begin{array}{l}\text { Etudiants / Futurs } \\
\text { enseignants FLE }\end{array}$ \\
\hline \multirow{4}{*}{ 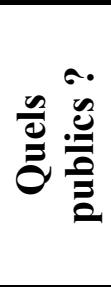 } & $\begin{array}{l}\text { Adolescents } \\
\text { en difficultés }\end{array}$ & $\mathbf{X}$ & & & \\
\hline & Migrants & & & & $\mathbf{X}$ \\
\hline & Adultes & & & & $\mathbf{X}$ \\
\hline & Détenus & & $\mathbf{X}$ & $\mathbf{X}$ & \\
\hline \multirow{4}{*}{ ن } & $\begin{array}{l}\text { Savoir } \\
\text { enseigner }\end{array}$ & $\mathbf{X}$ & & $\mathbf{X}$ & $\mathbf{X}$ \\
\hline & $\begin{array}{l}\text { Découvrir } \\
\text { l'univers } \\
\text { carcéral }\end{array}$ & & $\mathbf{X}$ & $\mathbf{X}$ & \\
\hline & $\begin{array}{l}\text { Enseigner à } \\
\text { un public en } \\
\text { difficulté }\end{array}$ & $\mathbf{X}$ & & & \\
\hline & $\begin{array}{l}\text { Agir face à } \\
\text { une situation } \\
\text { d'illettrisme }\end{array}$ & & & & $\mathbf{X}$ \\
\hline
\end{tabular}




\begin{tabular}{|c|c|c|c|c|}
\hline \multirow{3}{*}{ 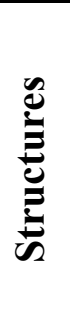 } & $\begin{array}{l}\text { Enseignement } \\
\text { primaire et } \\
\text { secondaire }\end{array}$ & $\mathbf{X}$ & & $\mathbf{X}$ \\
\hline & SEGPA & $\mathbf{X}$ & & \\
\hline & $\begin{array}{l}\text { Etablissement } \\
\text { pénitentiaire }\end{array}$ & $\mathbf{X}$ & $\mathbf{X}$ & $\mathbf{X}$ \\
\hline
\end{tabular}

\section{Quelle formation pour répondre aux besoins d'un public d'apprenants-détenus ?}

\subsection{Master FLE spécialisation « adultes détenus »}

Un Master DDL-FLE répond à une situation d'enseignement à un public adulte nonfrancophone. Certaines formations universitaires peuvent également être spécialisées dans l'enseignement du FLS à un public migrant. Toutefois, aucune formation ne propose l'enseignement du français langue seconde à un public dit « en difficulté », contrairement à une formation spécialisée délivrée par l’Education nationale.

Une unité d'enseignement sous forme d'option pourrait alors être proposée aux étudiants inscrits en Master DDL-FLE des universités françaises. Le contenu de ces unités d'enseignement apporterait des connaissances supplémentaires sur la pédagogie différenciée, le choix des supports en fonction du type de public, et pourrait comprendre des interventions de professionnels.

Il est toutefois compréhensible que de telles options n'existent pas actuellement dans les formations universitaires, dans la mesure où les structures concernées sont sous la tutelle de l'EN.

\subsection{Formation de l'EN spécialisation public adultes en difficulté}

Comme évoqué auparavant, les formations délivrées pour les enseignants de l'EN intervenant en milieu carcéral concernent principalement le contexte. Le problème majeur concerne le public. En effet, il s'agit, dans le contexte, d'un public adulte. Les enseignants intervenant en prison sont, pour la plupart, des enseignants du premier degré. Aucune formation spécialisée proposée par l'EN ne correspond aux attentes et aux besoins d'un adulte.

Bien que la formation CAPA-SH apporte des connaissances supplémentaires sur les besoins d'un apprenant en difficulté, elle ne répond pas au profil d'un apprenant adulte. Il serait donc judicieux que le Ministère de l'éducation propose aux enseignants une formation spécialisée pour l'enseignant à un public adulte. 


\subsection{CAPES FLE}

Etant donné que l'enseignement dans les établissements pénitentiaires français est régi par l'AP et l'EN, un enseignant titulaire d'un Master FLE ne peut y intervenir, bien que la discipline enseignée fasse partie de son champ de compétence. Il serait alors envisageable (et relèverait probablement d'une utopie) que le Ministère de l'éducation nationale reconnaisse enfin le français langue étrangère/seconde comme discipline à part entière, par le biais d'un CAPES FLE. Cette discipline est à l'heure actuelle peu valorisée par le Ministère, qui préfère affecter des enseignants de disciplines variées à des postes d'enseignement du FLE. Or, être locuteur francophone natif ne permet pas nécessairement d'enseigner sa langue.

De même que le FLE et l'alphabétisation sont confondus dans ce contexte d'enseignement particulier, les enseignants sont eux aussi concernés par cette nonreconnaissance de la discipline. Une formation permettant la titularisation d'un enseignant de FLE au sein de l'EN permettrait alors de résoudre ces difficultés : les enseignants intervenant en milieu carcéral seraient véritablement qualifiés pour l'enseignement d'une langue seconde, et les enseignements dispensés en prison correspondraient mieux aux profils des apprenants.

Toutefois, la création d'un concours du CAPES pour le champ du FLE ne fait aujourd'hui pas encore partie des préoccupations du Ministère. Néanmoins, la création d'une option FLE dans le CAPES de Lettres est proposée. Cela permettrait aux enseignants de lettres d'acquérir les compétences pour enseigner le français comme langue seconde. Une première étape est franchie, mais ne concerne toutefois que les enseignants ayant suivi un parcours littéraire. Qu'en est-il alors des nombreux enseignants de FLE issus d'une formation linguistique?

\section{Conclusion}

Examinons maintenant le cas du Québec. Dans cette province francophone du Canada, l'enseignement $\mathrm{du}$ français langue seconde est un enseignement reconnu par le Ministère de l'éducation. Il existe donc des formations universitaires permettant aux enseignants de FLS d'intégrer la fonction publique. Différentes formations proposent des spécialisations à des publics variés (migrants, étudiants, etc.). Bien qu'aucune formation ne réponde aux besoins des apprenants-détenus, le professeur de FLS acquiert suffisamment de connaissances sur la pédagogie à un public adulte et la didactique du français langue seconde. Une formation « de terrain » suffirait donc à ses enseignants pour intervenir en contexte carcéral. 
En France, la création d'une formation spécialisée «FLE/FLS » permettrait aux enseignants titulaires de l'EN d'être entièrement compétents dans une situation d'enseignement en milieu carcéral. Cette formation apporterait des connaissances complémentaires sur l'enseignement à un public adulte en difficulté et pour l'enseignement d'une langue seconde. Elle pourrait être basée sur des témoignages de professionnels et des stages pratiques. Enfin, à la suite de cette formation spécialisée, l'enseignant pourra recevoir une formation "pratique » délivrée par l'AP. Cette formation lui permettra de comprendre davantage le fonctionnement d'un établissement pénitentiaire, pour faire face à un public détenu.

\section{Bibliographie}

\section{L'enseignement en contexte carcéral}

Cavet, Christiane. « Vaincre l’illettrisme ». Economie \& Humanisme 363 (déc. 2012). 13.

Bailleau, Francis, Nathalie Gourmelon et Philip Milburn. «Les établissements privatifs de libertés pour mineurs : entre logiques institutionnelles et pratiques professionnelles ». Etudes et données pénales 112 (2012) : 285.

Febrer, Michel. Enseigner en prison : le paradoxe de la liberté pédagogique dans un univers clos. Paris : Harmattan, 2011.

Foucault, Michel. Surveiller et punir, Naissance de la prison. Paris : Gallimard, 1975.

Leterrier, Patrick. Et là vivent des hommes. Paris : Harmattan, 2007.

Milly, Bruno. «L'enseignement en prison: du poids des contraintes pénitentiaires à l'éclatement des logiques professionnelles ». Déviance et société (2004) : 57-79.

Salane, Fanny. «L'enseignement à distance en milieu carcéral, droit à l'éducation ou privilège : le cas des « détenus-étudiants ». Distances et savoirs 3 (2008) : 413-436.

\section{La formation des enseignants spécialisés}

Convention du 08/12/2011 entre le Ministère de l'éducation nationale et le Ministère de la Justice et des libertés.

«Orientations pédagogiques pour les enseignants généraux et professionnels adaptés dans le second degré ». Bulletin Officiel 18, 30/04/2009.

\section{Rapports d'activité}

«Les Centres éducatifs fermés, Rapport d'activité 2011 du Contrôleur général des lieux de privation de liberté (extraits) ». Journal du droit des jeunes 314, (avril 2012) : 18. 
«L'enseignement en milieu pénitentiaire : Rapport annuel sur l'année 2011 ». Direction de l’Administration Pénitentiaire, Pôle enseignement - PMJ3.

\footnotetext{
NOTES

${ }^{1}$ L'enseignement du premier degré regroupe les enseignements préélémentaires et élémentaires. L'enseignement du second degré regroupe les enseignements dispensés en collège, lycée général, lycée professionnels et technologiques.

${ }^{2}$ Le Bulletin Officiel définit les nouvelles mesures, lois ou décrets établis par le Ministère de l'Education Nationale français. Ils sont consultables en ligne sur le site du Ministère.
} 